\title{
USEFULNESS OF THE HIV DEMENTIA SCALE IN NIGERIAN PATIENTS WITH HIV/AIDS
}

\author{
Olubunmi A Ogunrin, $B S c, M B C h B, F W A C P$ \\ Emeka U Eze, $M B B S, F W A C P$ \\ Francis Alika, $M B B S$ \\ Department of Medicine, University of Benin Teaching Hospital, Benin City, Nigeria
}

\begin{abstract}
Objective. Information on the cognitive complications of HIV/AIDS from sub-Saharan Africa, where statistics on HIV are alarming, is sparse because of lack of validated cognitive tools. This study assessed the usefulness and predictive validity of the HIV Dementia Scale (HDS) as a screening tool in HIV-positive Nigerians.
\end{abstract}

Design. HIV-positive patients were randomly selected over a period of 2 months.

Setting. The HIV/AIDS outpatient clinic at the University of Benin Teaching Hospital, Benin City, Nigeria.

Subjects. Asymptomatic and symptomatic HIV-positive patients were compared with controls matched with regard to age, gender and level of education.

Outcome measures. Cognitive performances on the modified HDS.

Results. Performances on the HDS of 160 HIV-positive subjects (80 asymptomatic and 80 symptomatic) were compared with those of 80 HIV-negative controls. The mean HDS scores (maximum 12) were 10.78 (significant deviation (SD) 1.18) (HIV-negative subjects), 8.85 (SD 1.38) (HIV, asymptomatic) and 5.2 (SD 1.13) (HIV, symptomatic); $p<0.01$. The HDS was found to have sensitivity of $97.3 \%$, specificity of $80.4 \%$, accuracy of $91.9 \%$ and a positive predictive value of $91.4 \%$ and a negative predictive value of $93.2 \%$.

Conclusion. The HDS was shown to be a sensitive screening tool for patients with HIV/AIDS in sub-Saharan Africa, but it was insensitive to memory impairment in asymptomatic HIV-positive patients.

There has been considerable interest in the neuropsychological complications of HIV/AIDS in the last two decades. ${ }^{1,2} \mathrm{~A}$ range of HIV-related cognitive complications has been reported to include poor performance on tests of attention and concentration, movement and coordination, reaction time and mental flexibility. ${ }^{13,4}$ These HIV-associated neurocognitive deficits (HAND) manifest in their mild form as minor cognitive motor disorder (MCMD) and grossly as HIV-associated dementia (HAD). The annual incidence of $\mathrm{HAD}$ is $7 \%$ after development of AIDS $^{5}$ and it occurs in $20 \%$ of all HIV-infected persons, ${ }^{6}$ although prevalence rates in sub-Saharan Africa are higher, ranging between $16 \%$ and $54 \% .^{7-9}$ With an HIV prevalence of $5.8 \%$ among adults there are more than 3 million people living with HIV/AIDS (PLWHA) in Nigeria, more than in any other country in the world with the exception of South Africa and India. ${ }^{10-12}$

HIV-associated cognitive impairment may be a factor contributing to poor medication adherence in sub-Saharan Africa. With the increasing burden of disease there is clearly a need for a simple tool for rapid screening of cognitive functioning in HIV-infected persons. Efforts to develop appropriate screening techniques include the HIV Dementia Scale (HDS), ${ }^{13}$ a brief measure that has shown promise but lacks extensive independent evaluation. The HDS (especially the modified and international versions, which exclude a difficult-to-administer antisaccadic task) has been shown to be simpler to administer than most cognitive tests used in HIV patients and may be useful for screening of cognitive dysfunction in clinics with no neurologist or neuropsychologist on the staff, as it does not require special training. ${ }^{13,14}$ This attribute makes it appealing in the African setting, where it is likely to be applied by primary care providers because of the dearth of neuropsychologists in health care facilities. The present study examines the usefulness of the modified HDS in screening for cognitive deficits in a sample of HIV-positive adults in Nigeria. Dementia was diagnosed according to the universally accepted criteria in the Diagnostic and Statistical Manual for Mental Diseases, 4th edition (DSM-IV-TR). ${ }^{15}$ 


\section{PATIENTS AND METHODS}

A total of 160 antiretroviral therapy-naïve subjects with positive enzyme-linked immunosorbent assays for HIV were randomly selected (using a table of random numbers) from the HIV/AIDS clinic at the University of Benin Teaching Hospital, Benin City, Nigeria, between May and June 2006. Demographic data were obtained and the HDS was used to assess the patients' cognitive status after a detailed physical examination, including a comprehensive neuropsychiatric evaluation. The HIV-positive subjects were compared with 80 healthy seronegative subjects selected randomly from hospital staff and undergraduate students. Informed consent was obtained from the patients and the controls. The patients and the controls were matched with regard to age, gender and level of education.

The HIV-positive patients were categorised into two groups, asymptomatic and symptomatic, on the basis of their CD4+ T-lymphocyte counts $(<200$ cells/ $\mu$ for symptomatic) and the presence of HIV/AIDS-defining symptoms (unexplained fever - core body temperature $>37.2^{\circ} \mathrm{C}$ - for more than 4 weeks, diarrhoea for more than 4 weeks, unexplained weight loss of $>10 \%$ of previous body weight, and a generalised papular rash). We did not determine HIV clades (i.e. genetic subtypes of HIV groups) owing to lack of facilities, but some earlier studies have shown that the pattern of cognitive impairments may be affected by different clades in sub-Saharan Africa. ${ }^{16,17}$

Clinical neuropsychological evaluation including the identification of diagnostic features of dementia based on the DSM-IV-TR was conducted on all patients by a single neurologist (OAO) who was blind to the HDS rating. Deterioration in daily functioning was determined from information provided by family members living with the patients. They were questioned on the presence of the following cognitive symptoms: (i) memory disturbances (forgetfulness of new events, difficulty finding words or not knowing common facts) affecting daily activities; (ii) inability to cope with employment, academic demands and social activities that the patient could cope with before the onset of the illness; and (iii) deterioration in day-to-day functioning (i.e. difficulty in driving, shopping, handling money and self-care). Patients without functional deterioration, i.e. cognitive disturbances sufficient to interfere with day-to-day functioning, were not considered to have dementia.

Exclusion criteria included age under 18 years, already being on antiretroviral therapy, co-morbidity (diabetes mellitus, hypertension, epilepsy, hepatitis, intracranial disorders such as brain tumour, and other metabolic diseases), an inconclusive diagnosis, a major axis I psychiatric illness, a history of substance abuse, presence of clinical signs of cardiac failure, alcohol intake above $120 \mathrm{~g}$ per week or 13 units per week, a history of previous head injury with loss of consciousness, and anticholinergic medications.

\section{MEASURES}

The HDS was developed as a rapid screening test to assess for HAD. ${ }^{13}$ It is a paper-and-pencil neuropsychological instrument with objective subtests measuring psychomotor processing speed, verbal memory, constructional ability and executive function (response inhibition, set shifting) (Fig. 1). The score is based on performance for each subtest. This scale also exists in a modified form (eliminating the anti-saccadic subtest, which is often difficult to administer), and both the earlier and the modified versions have been validated for determining the presence and severity of HAD. ${ }^{12}$ The modified version was used in this study.

The modified scale has a maximum score of 12 , i.e. psychomotor speed score of 6 , verbal memory recall score of 4 , and construction task score of 2 . Details are outlined in Fig. 1. For the psychomotor speed and construction task, the subject is timed using a stopwatch and the appropriate score given as indicated in Fig. 1. The modified HDS was administered by one of the authors (EE), who had no prior knowledge of the functional status categorisation using the DSM-IV-TR.

\section{STATISTICAL ANALYSES}

Statistical analysis was done using Epi Info 2000 software (Centers for Diseases Control and Prevention, Atlanta, Ga). The age, gender and level of education of the three groups were analysed for significant differences using the chi-square test. The means of the performances of the HIV-positive patients were compared with those of the controls using the two-way analysis of variance. An F-test was used to determine the trend of linearity between cognitive performances and $\mathrm{CD} 4+$ levels. The level of significance was $p<0.05$. A receiver operating curve (ROC) was used to determine the cutoff score for HDS. The strength of association between cognitive disturbance and HIV seropositivity was determined with Mantel-Haenszel matched analysis and expressed as a likelihood ratio with 95\% confidence interval ( $\mathrm{Cl}$ ). The predictive value model using the $2 \times 2$ contingency table yielded the sensitivity, specificity and predictive values.

\section{RESULTS}

The demographic data for the subjects are set out in Table I. The majority were in the 21 - 40-year age range. Clinical characteristics of the HIV-positive symptomatic subjects are set out in Table II. Mean (SD) CD4+ Tlymphocyte counts for the controls, the asymptomatic HIV-positive patients and the symptomatic HIV-positive patients were 668 (SD 8.6), 286 (SD 7.4) and 102 (SD 10.8) cells/ $\mu \mathrm{l}$, respectively $(p<0.01)$. Mean (SD) total 


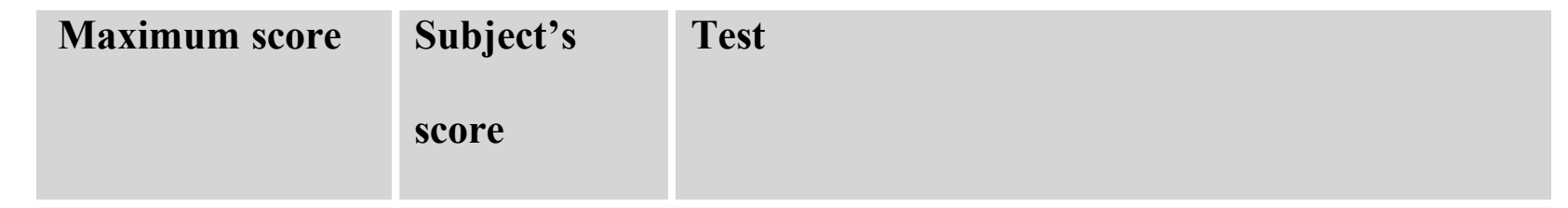

\section{Memory-Registration}

Give 4 words to recall and 1 second to say each. Then ask the patient all 4 after you have said them.

\begin{tabular}{|l|l|l}
\hline 6 & $\begin{array}{l}\text { Psychomotor speed } \\
\text { Ask patient to write the alphabet in uppercase letters } \\
\text { horizontally across the page (use back of questionnaire) and } \\
\text { record time } \ldots . . . \text { seconds. } \\
\mathbf{2} \mathbf{2 1} \mathbf{s e c}=\mathbf{6} ; \mathbf{2 1 . 1}-\mathbf{2 4} \mathbf{s e c}=\mathbf{5} ; \mathbf{2 4 . 1}-\mathbf{2 7} \mathbf{s e c}=\mathbf{4} ; \mathbf{2 7 . 1}-\mathbf{3 0} \\
\mathbf{s e c}=\mathbf{3} ; \mathbf{3 0 . 1}-\mathbf{3 3} \mathbf{s e c}=\mathbf{2} ; \mathbf{3 3 . 1} \mathbf{- 3 6}=\mathbf{1} ; \mathbf{3 6} \mathbf{s e c}=\mathbf{0}\end{array}$ \\
\hline 4 & $\begin{array}{l}\text { Memory }- \text { Recall } \\
\text { Ask for the } 4 \text { words from Memory }- \text { Registration test above. } \\
\text { Give } 1 \text { point for each correct word. For words not recalled, } \\
\text { prompt with a semantic clue. Give half a point for each } \\
\text { correct word after prompting. }\end{array}$ \\
\hline
\end{tabular}

2

\section{Construction}

Copy the cube below; record time ...... seconds

$$
<25 \mathrm{sec}=2 ; 25-35 \mathrm{sec}=1 ;>35 \mathrm{sec}=0
$$

Fig. 1. Modified HIV Dementia Scale.

HDS scores (maximum 12) for the controls, asymptomatic HIV-positive patients and symptomatic HIV-seropositive patients were 10.78 (SD 1.18), 8.85 (SD 1.38) and 5.2 (SD 1.13), respectively $(F=522.28, p<0.001)$. Details of the performances for the three categories are set out in Table III. There was a significant difference between the total scores for the normal subjects and the HIV-positive patients, irrespective of whether the patients were symptomatic or not. The asymptomatic HIV-positive patients performed better than the symptomatic patients $(t=20.13, p<0.001)$. Comparison of the memory scores did not reveal a significant difference between the performances of the controls and the asymptomatic HIV-positive patients, but the performance of the symptomatic HIV-positive patients was significantly poorer $(p<0.05)$.

The psychomotor speed of the HIV-positive subjects was significantly prolonged compared with the controls $(p<0.001)$ but the time taken to perform the construction task did not differ significantly between the controls and the asymptomatic seropositive patients $(p>0.05)$, although the construction task was performed poorly by the asymptomatic HIV-positive group (Table III). Deterioration in the performance of the HIVseropositive subjects as CD4+ T-lymphocyte counts decreased was observed. The $F$-test for linear trend showed this observation to be significant $(p<0.05)$, as outlined in Table IV.

A cut-off score of 9, obtained by ROC analysis, was used to determine the sensitivity, specificity and predictive values of the HDS. The HDS total scores were below the cut-off score of 9 in 109 HIV-seropositive patients $(68.1 \%)$, but $41(25.6 \%)$ of the patients had no features of functional deterioration and their HDS total scores were above 9. The total HDS scores were normal (i.e. above the cut-off score of 9) in 10 patients with functional deterioration, while 4 patients had no functional deterioration but had abnormal total HDS scores (Table V). This implies false-positive and false-negative rates of $8.6 \%$ and $6.8 \%$, respectively. The likelihood ratio for cognitive disturbance in HIV-positive patients based on modified HDS scores in the presence of functional deterioration was 41 (95\% Cl $13-138)$. This implies that patients with clinical evidence of dementia based on cognitive symptoms sufficient to interfere with functional abilities are approximately 40 times more likely to have a score of $<9$ on the modified HDS. The sensitivity of the modified HDS was $97 \%$, the specificity was $80 \%$, the positive predictive value was $91 \%$ and the negative predictive value was 93\% (overall accuracy, 92\%). 


\section{TABLE I. DEMOGRAPHIC DATA OF THE STUDY SUBJECTS}

\begin{tabular}{|c|c|c|c|}
\hline & Controls $(N=80)$ & Asymptomatic HIV positive $(N=80)$ & Symptomatic HIV positive $(N=80)$ \\
\hline \multicolumn{4}{|l|}{ Gender } \\
\hline Male & 32 & 32 & 34 \\
\hline Female & 48 & 48 & 46 \\
\hline$\chi^{2}$ & 0.104 & & \\
\hline$p$ & 0.95 & & \\
\hline \multicolumn{4}{|c|}{ Age groups (yrs) } \\
\hline$\leq 20$ & 6 & 4 & 6 \\
\hline $21-30$ & 25 & 26 & 26 \\
\hline $31-40$ & 27 & 28 & 26 \\
\hline $41-50$ & 14 & 14 & 15 \\
\hline$\geq 50$ & 8 & 8 & 7 \\
\hline $\bar{\chi}^{2}$ & 0.734 & & \\
\hline$\tilde{p}$ & 0.99 & & \\
\hline Age range (yrs) & $8-58$ & $20-64$ & $18-56$ \\
\hline \multicolumn{4}{|c|}{ Level of education } \\
\hline Primary & 18 & 19 & 18 \\
\hline Secondary & 37 & 38 & 38 \\
\hline Tertiary & 25 & 23 & 24 \\
\hline$\chi^{2}$ & 0.137 & & \\
\hline$p$ & 0.99 & & \\
\hline
\end{tabular}

TABLE II. CLINICAL CHARACTERISTICS OF THE SYMPTOMATIC HIV-POSITIVE SUBJECTS

\begin{tabular}{lcc} 
Symptom & Frequency $(M)$ & $\%$ \\
\hline Unexplained fever $\left(>37.2^{\circ} \mathrm{C}\right.$ for $>4$ wks) & 72 & 90 \\
Unexplained weight loss $(>10 \%$ of previous body weight) & 76 & 95 \\
Persistent diarrhoea $(>4$ wks) & 62 & 78 \\
Generalised papular rash & 48 & 60 \\
Generalised herpes zoster & 18 & 23 \\
Clinical/radiological signs of pulmonary tuberculosis & 54 & 68 \\
Unexplained anaemia (haematocrit $<30 \%$ or haemoglobin<9 g/dl) & 68 & 85 \\
\hline
\end{tabular}

\section{DISCUSSION}

The epidemiology of HIV-associated cognitive impairment in Africa is poorly understood. Vaguely defined criteria for cognitive impairment and assessment tools that are inadequate and lack validation, coupled with absence of normative population data, have limited proper study of cognitive impairment in HIV-positive populations in these settings. Although the clinical presentation of dementia may vary, depending on the causation, the diagnostic features are constant as set out in the DSM-IV-TR. The performance of an individual in the cognitive subtests may therefore reflect the degree of interference with performing similar tasks in daily life, although this is not invariable, as a poor cognitive score does not always infer functional incapacity. By comparing the performances of HIV-positive patients with controls, we assessed the differences in their abilities in these cognitive domains.

This study showed the usefulness and predictive validity of the modified HDS as a screening cognitive tool in Nigerian patients. The findings applied to subjects who met the DSM-IV diagnostic criteria for dementia and were also reported by family members to display func- tional deterioration in performance of daily activities. The modified HDS revealed deficits in all the tested cognitive domains in the symptomatic HIV-positive patients. Although the modified HDS showed impairment in cognitive abilities of the asymptomatic HIV-positive patients compared with the controls, the memory performances were similar. The predictive values, sensitivity and specificity values obtained from this study are comparable to those obtained by earlier authors, who used cut-off scores of 9 and 7.5..$^{18,19}$ The memory scores and the time taken to perform the construction task did not differ significantly between controls and asymptomatic HIV-positive patients. A similar observation was reported by Smith et $a l^{20}$ These findings corroborate earlier reports of a low prevalence of memory impairment among asymptomatic HIV-positive patients. ${ }^{4,21,22}$

The total scores showed the ability of the modified HDS to demonstrate the presence of cognitive impairments in HIV/AIDS, and it is therefore likely to be especially useful for population-based studies. It clearly demonstrated the presence of psychomotor retardation in the HIV-positive subjects, as has been reported by others. ${ }^{1,5,14}$ The $68.1 \%$ prevalence of HAD observed in this study using the modified HDS is high. This figure 
TABLE III. HIV DEMENTIA SCALE SCORES FOR THE SUBJECTS

\begin{tabular}{|llll|} 
& Controls & Asymptomatic HIV positive & Symptomatic HIV positive \\
\hline Memory (mean SD)) & $3.68(0.15)$ & $3.56(0.64)$ & $1.82(1.84)$ \\
& & $(p>0.05)^{*}$ & $(p<0.001)^{\dagger}$ \\
Construction (mean (SD)) & $1.96(0.20)$ & $1.23(0.62)$ & $0.84(0.71)$ \\
& & $(p<0.05)$ & $(p<0.001$ \\
Time taken for task & $9.84(5.84)$ & $10.12(3.46)$ & $24.21(6.43)$ \\
(s) (mean (SD)) (range) & $(2-28)$ & $(4-32)$ & $(16-56)$ \\
& & $(p>0.05)$ & $(p<0.001)$ \\
Psychomotor speed (mean (SD)) & $5.14(0.77)$ & $4.06(1.72)$ & $2.54(1.46)$ \\
& & $(p<0.01)$ & $(p<0.001)$ \\
Time taken for task & $15.9(4.59)$ & $19.32(6.72)$ & $39.2(2.44)$ \\
(s) (mean (SD)) (range) & $(10-36)$ & $(11-48)$ & $(26-54)$ \\
& & $(p<0.001)$ & $(p<0.001)$ \\
Total score & $10.78(1.18)$ & $8.85(1.38)$ & $5.2(1.13)$ \\
& & $(p<0.001)$ & $(p<0.001)$ \\
& & & \\
\hline *Levels of significance for comparison between mean performances of the controls and asymptomatic HIV-positive subjects. & \\
\hline tevels of significance for comparison between mean performances of the controls and symptomatic HIV-positive subjects. &
\end{tabular}

TABLE IV. THE COMPARISON OF COGNITIVE PERFORMANCE WITH CD4+ LEVELS USING THE F-TEST FOR LINEAR TREND

\begin{tabular}{|llll|}
\hline Cognitive domains & Slope $\left(r^{2}\right)$ & $F$-values & $p$-values \\
\hline Memory & $-0.98(0.33)$ & 120.75 & $p<0.0001$ \\
Construction & $-0.41(0.26)$ & 86.90 & $p<0.0001$ \\
Time taken & $7.19(0.47)$ & 283.45 & $p<0.001$ \\
Psychomotor speed & $-1.15(0.31)$ & 111.70 & $p<0.001$ \\
Time taken & $9.15(0.64)$ & 556.76 & $p<0.001$ \\
$\quad$ Total & $-3.62(0.65)$ & 579.11 & $p<0.001$ \\
\hline
\end{tabular}

\begin{tabular}{|c|c|c|c|c|}
\hline & $\begin{array}{l}\text { Symptomatic HIV+ } \\
(N=80)\end{array}$ & $\begin{array}{l}\text { Asymptomatic HIV+ } \\
(N=160)\end{array}$ & $\begin{array}{l}\text { HIV+ subjects } \\
(N=80)\end{array}$ & $\begin{array}{l}\text { Controls HIV- } \\
(N=80)\end{array}$ \\
\hline \multicolumn{5}{|l|}{$\begin{array}{l}\text { Functional status* } \\
(N(\%))\end{array}$} \\
\hline Deterioration & $80(100.0)$ & 39 (48.75) & 119 (74.38) & NA \\
\hline Still coping & $0(0)$ & $41(51.25)$ & $41(25.62)$ & NA \\
\hline $\begin{array}{l}\text { HDS total score } \\
\text { (mean (SD)) } \\
\text { (maximum 12) }\end{array}$ & $5.2(1.13)$ & $8.85(1.38)$ & $7.03(1.64)$ & $10.78(1.18)$ \\
\hline Normal $(N(\%))$ & $4(5.0)$ & 47 (58.75) & $51(31.87)$ & $78(97.5)$ \\
\hline Abnormal $^{+}(N(\%))$ & $76(95.0)$ & $33(41.25)$ & $109(68.13)$ & $2(2.5)$ \\
\hline$\chi^{2} 84.94$ & \multicolumn{4}{|l|}{$p<0.001^{\ddagger}$} \\
\hline \multicolumn{5}{|c|}{$\begin{array}{l}\text { *Based on deterioration of functional abilities of patients (as expressed by family members living with patients). } \\
\text { +HDS cut-off score obtained by receiver operating characteristics analysis (cut-off score of } 9 \text {; abnormal score }<9 \text { ). } \\
\text { F Mantel-Haenszel matched analysis. } \\
\text { NA = not applicable. }\end{array}$} \\
\hline
\end{tabular}

is higher than rates of 16 - 54\% reported by other authors in Africa ${ }^{7-9}$ but similar to the $64.3 \%$ prevalence of cognitive impairment in a Nigerian population with asymptomatic mild HIV infection. ${ }^{23}$ This may be due to HIV clade diversity and the differences in the sensitivity of neurocognitive assessment tools utilised in these studies. More recently studies have demonstrated the presence and pattern of cognitive impairments in black Africans with HIV/AIDS, but most of these studies used either complex psychometric ${ }^{21,24}$ or computerised cognitive tests, ${ }^{22,25}$ which are difficult to administer in most rural clinical settings and cumbersome for community-based research in sub-Saharan Africa.
The prevalence data available for HIV-associated cognitive impairment in Africa represent rates in people presenting to tertiary care centres and are unlikely to reflect rates in the general population. The level of education of research subjects affects performance on the HDS (a higher level of education improving cognitive performance), but the effects of age and gender remain inconclusive. ${ }^{3,19}$ The HDS has been validated in subcortical cognitive impairment, the pattern of impairment observed in HIV-associated neurocognitive deficits. ${ }^{19}$ The modified HDS requires no computer. It is a pen-and-paper cognitive instrument that can easily be administered in rural clinic settings. It can be 
administered by health care providers who are nonprofessionals but have received instruction in its application. It is therefore suitable for community-based research in low-income developing countries. It has acceptable false-negative and false-positive rates. On the basis of our findings we believe it to be a useful screening tool for determining the baseline cognitive abilities of patients with HIV/AIDS and recommend its use for monitoring the response of patients with HIVassociated cognitive impairment who are on highly active antiretroviral therapy (HAART), as an earlier study has demonstrated improvement in neurocognitive and functional performance in HIV-positive individuals on HAART in sub-Saharan Africa. ${ }^{26}$

In conclusion, this study suggests the usefulness of the HDS as a screening tool for the assessment of cognitive abilities of patients with HIV/AIDS in sub-Saharan Africa. The sensitivity, specificity and predictive values compared favourably with those obtained among patients in developed countries. It is, however, limited by its inability to detect significant memory impairment in asymptomatic HIV-seropositive patients.

\section{REFERENCES}

1. Heaton RK, Grant I, Butters N, et al. Neuropsychology of HIV infection at different disease stages - HIV Neurobehavioural Research Center. J Int Neuropsychol Soc 1995; 1: 231-251.

2. McArthur JC, Sacktor N, Selnes 0 . Human immunodeficiency virus-associated dementia. Semin Neurol 1999; 19: 129-150.

3. Richardson MA, Morgan EE, Vielhauer MJ, et al. Utility of the HIV dementia scale in assessing risk for significant HIV-related cognitive-motor deficits in a high-risk urban adult sample. AIDS Care 2005; 17(8): 1013-1021.

4. Selnes OA. Memory loss in persons with HIV/AIDS: assessment and strategies for coping. AIDS Reader 2005; 15: 289-294.

5. Almeida SM de, Letendre $S$, Ellis R. Human Immunodeficiency virus and the central nervous system. Braz J Infect Dis 2006; 10(1): 41-50.

6. McArthur JC, Hoover DR, Bacellar H. Dementia in AIDS patients: incidence and risk factors. Neurology 1993; 43: 2245-2252.
7. Howlett, WP, Nkya WM, Mmuni KA, et al. Neurological disorders in AIDS and HIV disease in the northern zone of Tanzania. AIDS 1989; 3(5): 289-296.

8. Sacktor NC, Wong M, Nakasujja N, et al. The International HIV Dementia Scale: a new rapid screening test for HIV dementia. AIDS 2005; 19: 1367-1374.

9. Wong MH, Robertson $K$, Nakasujja N, et al. Frequency of and risk factors for HIV dementia in an HIV clinic in sub-Saharan Africa. Neurology 2007; 68(5): 350355.

10. UNAIDS report on the global HIV/AIDS epidemic. Joint United Nations Program on HIV/AIDS, Geneva. http://www.unaids.org/en/Regions_Countries/Regions/ SubSaharanAfrica.asp (accessed 5 July 2004).

11. Sani MU, Mohammed AZ, Adamu B, et al. AIDS mortality in a tertiary institution: a four-year review. J Natl Med Assoc 2006; 98: 862-866.

12. Federal Ministry of Health Nigeria/National Action Committee on HIV/AIDS. 2006 National HIV Sero-prevalence Sentinel Survey: Technical Report. Abuja: Federal Ministry of Health, 2007

13. Power $C$, Selnes $O A$, Grim JA, et al. HIV Dementia Scale: a rapid screening test. $J$ Acquir Immune Defic Syndr Hum Retrovirol 1995; 8(3): 273-278.

14. von Giesen HJ, Haslinger BA, Rohe $\mathrm{S}$, et al. HIV dementia scale and psychomotor slowing - the best methods in screening for neuro-AIDS. J Neuropsychiatry Clin Neurosci 2005; 17(2): 185-191.

15. American Psychiatric Association. Diagnostic and Statistical Manual of Mental Disorders. 4th ed., text revised. Washington, DC: American Psychiatric Association, 2000.

16. Clifford DB, Mitike MT, Mekonnen Y, et al. Neurological evaluation of untreated human immunodeficiency virus infected adults in Ethiopia. J Neurovirol 2007; 13(1): 67-72.

17. Rolfe M. HIV-2 and its neurological manifestations. S Afr Med J 1994; 84: 503505.

18. Davis HF, Skolasky RL Jr., Selnes OA, et al. Assessing HIV-associated dementia: modified HIV dementia scale versus the Grooved Pegboard. AIDS Reader 2002 12(1): 32-33.

19. van Harten B, Courant MN, Scheltens $P$, et al. Validation of the HIV Dementia Scale in an elderly cohort of patients with subcortical cognitive impairment caused by subcortical ischemic vascular disease or a normal pressure hydrocephalus. Dement Geriatr Cogn Disord 2004; 18(1): 109-114.

20. Smith CA, van Gorp WG, Ryan ER, et al. Screening subtle HIV-related cognitive dysfunction: the clinical utility of the HIV dementia scale. J Acquir Immune Defic Syndr Hum Retrovirol 2003; 33: 116-118.

21. Odiase $F$, Ogunnin 0 , Ogunniyi A. Effect of progression of disease on cognitive performance in HIV/AIDS. J Natl Med Assoc 2006; 98: 1260-1262.

22. Ogunrin 0, Odiase F. Motor speed and reaction time in HIV/AIDS patients: a casecontrol study. African Journal of AIDS Research 2006; 5(3): 217-220.

23. Salawu FK, Bwala SA, Wakil MA, et al. Cognitive function in HIV-seropositive Nigerians without AIDS. J Neurol Sci 2008; 267: 142-146.

24. Birbeck GL. Human immunodeficiency virus dementia patients in Africa. How many? Who cares? And where to from here? J Neurovirol 2005; 11 (Suppl. 36): 30-33

25. Miller EN, Satz P, Visscher BV. Computerized and conventional neuropsychological assessment of HIV-infected homosexual men. Neurology 1991; 41: 1608-1616.

26. Sacktor N, Nakasujija N, Skolasky $\mathrm{R}$, et al. Antiretroviral therapy improves cognitive impairment in HIV+ individuals in sub-Saharan Africa. Neurology 2006; 67(2): $311-314$ 